\title{
Brefeldin A-inhibited Guanine Exchange Factor 2 Regulates Filamin A Phosphorylation and Neuronal Migration
}

\author{
Jingping Zhang, ${ }^{1 \star}$ Jason Neal, ${ }^{1 \star}$ Gewei Lian, ${ }^{1}$ Bingxing Shi, ${ }^{2}$ Russell J. Ferland, $, 2,3$ and Volney Sheen ${ }^{1}$ \\ ${ }^{1}$ Department of Neurology, Beth Israel Deaconess Medical Center, Boston, Massachusetts 02115, ${ }^{2}$ Department of Biology, Center for Biotechnology and \\ Interdisciplinary Studies, Rensselaer Polytechnic Institute, Troy, New York 12180, and ${ }^{3}$ Department of Neurology, Center for Neuropharmacology and \\ Neuroscience, The Albany Medical College, Albany, New York 12208
}

Periventricular heterotopia $(\mathrm{PH})$ is a human malformation of cortical development associated with gene mutations in ADP-ribosylation factor guanine exchange factor 2 (ARFGEF2 encodes for Big2 protein) and Filamin A (FLNA). PH is thought to derive from neuroependymal disruption, but the extent to which neuronal migration contributes to this phenotype is unknown. Here, we show that Arfgef2 null mice develop PH and exhibit impaired neural migration with increased protein expression for both FlnA and phosphoFlnA at Ser2152. Big2 physically interacts with FlnA and overexpression of phosphomimetic Ser2512 FLNA impairs neuronal migration. FlnA phosphorylation directs FlnA localization toward the cell cytoplasm, diminishes its binding affinity to actin skeleton, and alters the number and size of paxillin focal adhesions. Collectively, our results demonstrate a molecular mechanism whereby Big2 inhibition promotes phosphoFlnA (Ser2152) expression, and increased phosphoFlnA impairs its actin binding affinity and the distribution of focal adhesions, thereby disrupting cell intrinsic neuronal migration.

\section{Introduction}

Neuronal progenitors migrate from the ventricular zone (VZ) into the cortical plate (CP) during brain development (Nadarajah and Parnavelas, 2002). The initial step in neuronal migration involves the coordinated extension of a leading process (through assembly and disassembly of focal adhesions), followed by translocation of the cell soma through a forward actin flow (Faux and Parnavelas, 2007). Local disruption of actin along the leading process disrupts somal translocation and migration (He et al., 2010). The extension of the leading edge is also mediated by the addition of new membrane at specific sites along the growing tip, thereby dictating a particular direction of migration. The exocyst complex oversees this process by directing the polarized delivery of the membrane and vesicles to preferential sites along the leading edge of migratory cells (Letinic et al., 2009).

The brefeldin A (BFA)-sensitive Sec7 guanine exchange protein 2 (BIG2) is a guanine exchange factor (GEF) that regulates vesicle budding from the Golgi and endosomal membrane com-

Received March 4, 2012; revised July 16, 2012; accepted July 21, 2012.

Author contributions: J.Z., J.N., and V.S. designed research; J.Z., J.N., and G.L. performed research; B.S. and R.J.F. contributed unpublished reagents/analytic tools; J.Z., J.N., and V.S. analyzed data; J.Z., J.N., and V.S. wrote the paper.

*J.Z. and J.N. contributed equally to this work.

This work was supported in part by National Institutes of Health Grants MH71801 (R.J.F.) and HD054347 and NS063997-01 (V.S.), the Empire State Stem Cell Fund through New York State Department of Health Contract C024324 (R.J.F., V.S.), March of Dimes Foundation Grant 5-FY09-29 (R.J.F.), and the Massachusetts' Alzheimer's Disease Research Center (V.S.). We thank Drs. C. Walsh, T. Stossel, K. Nakayama, J. Flanagan, J. Bonifacino, J. Blenis, and F. Nakamura for providing the valuable reagents specified in Materials and Methods. We appreciate the thoughtful comments from Drs. J. Flanagan, D. Van Vactor, and V. Hsu.

Correspondence should be addressed to Volney Sheen, Department of Neurology, Beth Israel Deaconess Medical Center, 330 Brookline Avenue, Boston, MA 02115. E-mail: vsheen@bidmc.harvard.edu.

DOI:10.1523/JNEUROSCI.1063-12.2012

Copyright $\odot 2012$ the authors $\quad 0270-6474 / 12 / 3212619-11 \$ 15.00 / 0$ partments. The nature of the protein cargo trafficked by BIG2 is not completely known, but deficiencies in BIG2 function have been shown to disrupt the localization of adhesion proteins (Achstetter et al., 1988; Sheen et al., 2004; Jones et al., 2005; D’Souza-Schorey and Chavrier, 2006). Moreover, intraventricular injection of BFA (BIG2 inhibitor) leads to impaired migration of early neural progenitors, alters localization of $\beta$-catenin, and periventricular heterotopia (PH) formation (Ferland et al., 2009). Loss of neuroependymal integrity has been shown previously to impair glial-guided neuronal migration with disruption of the radial glial scaffolding. However, $\beta$-catenin is also expressed along the leading edge of migratory cells, suggesting a potential trafficking defect of $\beta$-catenin within the radial glia cells (Jones et al., 2008). These observations raise the possibility that Big2 might intrinsically regulate neuronal migration into the CP.

$\mathrm{PH}$ is a congenital malformation of cortical development attributable to human mutations in two genes, Filamin A (FLNA) and ADP-ribosylation factor guanine exchange factor 2 (ARFGEF2) (Fox and Walsh, 1999; Sheen et al., 2004). This disorder is characterized by ectopic nodules of neurons that fail to migrate from the VZ into the cortex ( $\mathrm{Lu}$ and Sheen, 2005). The underlying molecular mechanism by which Big2 regulates cortical development and gives rise to this neuropathological phenotype is poorly understood. Here, we show that Arfgef2 $2^{-1-}$ mice can develop PH, indicative of disrupted neuronal migration. This defect in migration occurs through both non-cell-autonomous and cellautonomous mechanisms. Big2 physically interacts with FlnA, and these proteins are localized to both the perinuclear region and cell membrane when coexpressed. Big2 suppression leads to increased expression levels of both FlnA and phosphoFlnA (Ser2152). The resultant overexpression of phosphomimetic mutant FlnA (Ser2152), more so than phosphodeficient FlnA 
(Ser2152), leads to impaired neuronal migration. Last, phosphorylation of FlnA at Ser2152 causes redistribution of FlnA to the cytoplasm and alters paxillin containing focal adhesion number and size. These observations suggest that the impaired neuronal migration, seen with loss of Big2, occurs directly through its interaction with FlnA and, likely, indirectly through consequent FlnA-dependent effects on the actin-focal adhesion cytoskeletal machinery.

\section{Materials and Methods}

Mice. Generation of the Arfgef2 targeting vector and the Arfgef2 knockout strain was performed by inGenious Targeting Laboratory. Briefly, a $16.9 \mathrm{~kb}$ fragment of genomic DNA from a positively identified C57BL/6 BAC clone (RP23:216L18) containing the Arfgef2 locus was subcloned and used to construct the targeting vector. A $6 \mathrm{~kb}$ long homology arm of intronic sequence containing exon 6 of Arfgef 2 was the $3^{\prime}$ arm of the targeting vector (after the $3^{\prime}$ end of the Lox/FRT-Neo cassette). A $2 \mathrm{~kb}$ short homology arm of intronic sequence upstream of exon 2 of Arfgef2 was the $5^{\prime}$ arm of the targeting vector (preceding the $5^{\prime}$ end of the Lox/ FRT-Neo cassette) (see Fig. 1 A). The Lox/FRT-Neo cassette was used to replace a genomic region that spans exons $2-5$ in the same direction as the Arfgef2 gene. After electroporation of the Arfgef2 targeting vector into BA1 (C57BL/6 × 129/SvEv) hybrid embryonic stem cells, recombinant clones were screened by PCR. Positive recombinant ES cells were then microinjected into C57BL/6 blastocysts. Resulting chimeras with a high percentage agouti coat color were mated to wild-type (WT) C57BL/6 mice to generate F1 heterozygous offspring. Tail DNA was analyzed to confirm germ-line transmission. Arfgef $2^{+/-}$mice were bred to produce Arfgef $2^{+/+}$and Arfgef2 $2^{-/-}$mice for analysis. Mice were maintained on a normal $12 \mathrm{~h}$ light/dark cycle with unlimited access to food and water. Western blot analysis confirmed that Arfgef $2^{-1-}$ mice had no detectable Big2 protein expression (see Fig. $1 B$ ). All mouse procedures were performed under approval from the Institutional Animal Care and Use Committees of Harvard Medical School in accordance with the National Institutes of Health Guide for the Care and Use of Laboratory Animals. Sixty-two mouse embryos at embryonic day 18.5 (E18.5) of either sex were used for evaluation of the null mouse phenotypes.

Coimmunoprecipitation and yeast two-hybrid assays. For coimmunoprecipitation, human neuroblastoma (CHP100) cells were lysed in 50 mu Tris-HCl buffer ( $\mathrm{pH} 7.5$ ) containing $150 \mathrm{~mm} \mathrm{NaCl}, 3 \mathrm{~mm} \mathrm{KCl,} 1 \mathrm{~mm}$ EDTA, and $1 \%$ Triton X-100 with a mixture of protease inhibitors. The supernatant was incubated overnight at $4^{\circ} \mathrm{C}$ with antibodies against GFP (Invitrogen), FLNA (Novocastra), or BIG2 (Sheen et al., 2004). The precipitated immunocomplex was immobilized on protein A/G-Sepharose beads, and the target proteins were detected by Western blotting.

The fragments encompassing the multiple receptor binding domain of FLNA (amino acids 2167-2648, bait A) and N terminus of BIG2 (amino acids $1-654$, bait B) were used as bait. N-terminal (amino acids 1-654) and C-terminal (amino acids 786-1452 and 1120-1785) fragments of BIG2 were used as prey for yeast two-hybrid assays. All pNLex and pJG45 fusion constructs were transformed into the yeast EGY48 strain. The positive clones were grown under the proper selection conditions, and positive hybrid interactions were determined using a $\beta$-galactosidase assay (Sheen et al., 2002).

Bromodeoxyuridine injection and in utero electroporation. E14.5 pregnant WT and Arfgef2 $2^{-/-}$dams were injected once intraperitoneally with bromodeoxyuridine (BrdU) (50 mg/kg), killed at E18.5, and sectioned for immunohistochemistry. A total of six embryos of either sex were used for BrdU study. In utero electroporations were performed using E14.5E15.5 pregnant dams. Mice were deeply anesthetized with ketamine/ xylazine. A total of 1.0-3.0 $\mu \mathrm{l}$ of DNA and GFP mixture (2:1 ratio with $0.05 \%$ fast green) was injected into either lateral ventricle using a beveled pipette, and five pulses at $40 \mathrm{~V}$ with a $50 \mathrm{~ms}$ duration were delivered at $1 \mathrm{~s}$ intervals. The embryos were killed $72 \mathrm{~h}$ later, fixed in $4 \% \mathrm{PFA}$, and sectioned on a cryostat at $20 \mu \mathrm{m}$. The cortex was divided into the proliferating zone (PZ) (ventricular and subventricular zones), intermediate zone (IZ), and CP using nuclear staining. The percentages of positive GFP and BrdU cell somas found within the PZ, IZ, or CP or with respect to the ventral or dorsal halves of the $\mathrm{CP}$ was scored. At least six sections from each embryo were scored, and a total of four embryos of either sex for each group were used in this study. Significance was determined using a paired Student's $t$ test.

Plasmids and cell transfection. FLNA cDNAs were provided by the Stossel laboratory (Brigham and Women's Hospital, Boston, $\mathrm{MA}$ ) to generate all FLNA constructs. ARFGEF2 cDNA and the HA-Arfgef 2 construct for subcloning were provided by the Nakayama laboratory (Kyoto University, Kyoto, Japan). FLNA phosphomimetic and phosphodeficient Ser2152 were kind gifts from F. Nakamura (Stossel laboratory). Control and ARFGEF2 siRNA oligonucleotides were purchased from Ambion (Silencer Negative control catalog \#4611 and ARFGEF2 siRNA catalog \#16708A) and Sigma (Arfgef2 siRNA catalog \#SASI_Mm02_00300542, \#SASI_Mm02_00300544, and \#SASI_Mm02_00300543), and the knockdown efficiency was verified by Western blotting.

HEK-293, CHP100, and Neuro2a cells (purchased from American Type Culture Collection) were grown in DMEM (Invitrogen) and Eagle's MEM (American Type Culture Collection catalog \#30-2003) supplemented with $10 \% \mathrm{v} / \mathrm{v}$ fetal bovine serum (FBS). M2 cells (a gift from Dr. T. Stossel) were maintained as described previously (Vadlamudi et al., 2002). Neuronal progenitors were isolated from E14.5 mouse cortices and grown as neurospheres in neural progenitor growth medium as described previously (Feng et al., 2006). Mouse fibroblast (MEF) cells were isolated from E14.5 and cultured in DMEM with 10\% FBS. All transfections were performed using Lipofectamine 2000 (Invitrogen) or TransFectin (Bio-Rad) according to the protocol of the manufacturer.

Immunohistochemistry and immunocytochemistry. Timed (E18.5) embryos (six of either sex) were surgically removed from pregnant mothers according to Institutional Animal Care and Use Committee guidelines. The frozen and paraffin sections were performed according to previously described procedures (Ferland et al., 2006). H\&E staining was performed according to previously described procedures (Ferland et al., 2009). For immunohistochemistry, sections were blocked in PBS containing 5\% donkey serum, incubated overnight using the appropriate primary antibodies against Tbr1, Tbr2, and BrdU (Abcam), doublecortin (courtesy of Dr. Christopher Walsh, Children's Hospital, Boston, MA), nestin (Developmental Studies Hybridoma Bank), and GFP (Invitrogen). The following day, tissue sections were washed and treated with the appropriate secondary antibody for $1 \mathrm{~h}$. For immunocytochemistry, cells were blocked with $3 \%$ goat serum containing $0.25 \%$ Triton X-100 and stained for primary antibodies against Myc (Sigma), phosphoFLNA Ser2152 (gift from F. Nakamura), paxillin (BD Transduction Laboratories), and FLNA (Santa Cruz Biotechnology). All secondary antibodies and phalloidin (Invitrogen) were incubated at room temperature for $1 \mathrm{~h}$. Fluorescent microscopic images were prepared using an Olympus AX70 microscope with the SNAP microphotographic system. Confocal images were obtained using a Carl Zeiss laser scanning microscope.

Tissue dissection, Triton X-100 fractionation, and Western blot. Tissues (liver, heart, brain, and skin) from 20 of either sex at E16.5 or postnatal day 0 (P0) were removed from WT or Arfgef $2^{-1-}$ mouse pups and then immediately homogenized in lysis buffer [50 mM Tris-HCl, pH 7.5, 150 mм NaCl, 2 mм EDTA, 1\% Triton X-100, complete protease inhibitor cocktail (Roche), and phosphatase inhibitor cocktail (Sigma)] using a Dounce homogenizer. CHP100 cells were serum starved overnight in DMEM and either left untreated or treated with $10 \mu \mathrm{M}$ forskolin (Fisher BioReagent; Thermo Fisher Scientific) or H89 (PKA inhibitor from Tocris Bioscience) for $10 \mathrm{~min}$ on the following day, and cell lysates were obtained in lysis buffer. For fractionation, treated cells were incubated on ice with extraction buffer (10 mM HEPES, pH7.4, 2 mM EDTA, 320 mM sucrose, $0.5 \%$ Triton X-100, and a mixture of protease and phosphatase inhibitor) for $10 \mathrm{~min}$. The soluble and insoluble fractions were separated by centrifugation at $15,000 \mathrm{rpm}$ at $4^{\circ} \mathrm{C}$ for $1 \mathrm{~h}$. All samples were separated on SDS-PAGE, and the proteins were detected by Western blotting using antibodies against phosphoFlnA (Ser2152) and FLNA (Epitomics), Myc (Sigma), tubulin (Millipore), actin (Santa Cruz Biotechnology), and GST (GE Healthcare). The ratio of immunoblotting signaling intensity was measured using NIH ImageJ software, and the results were calculated from four to five independent experiments.

Transwell assay. CHP100 cells transfected with the indicated DNA plasmids were plated at $5 \times 10^{4}$ cells in 24 -well inserts (BD Biosciences). 

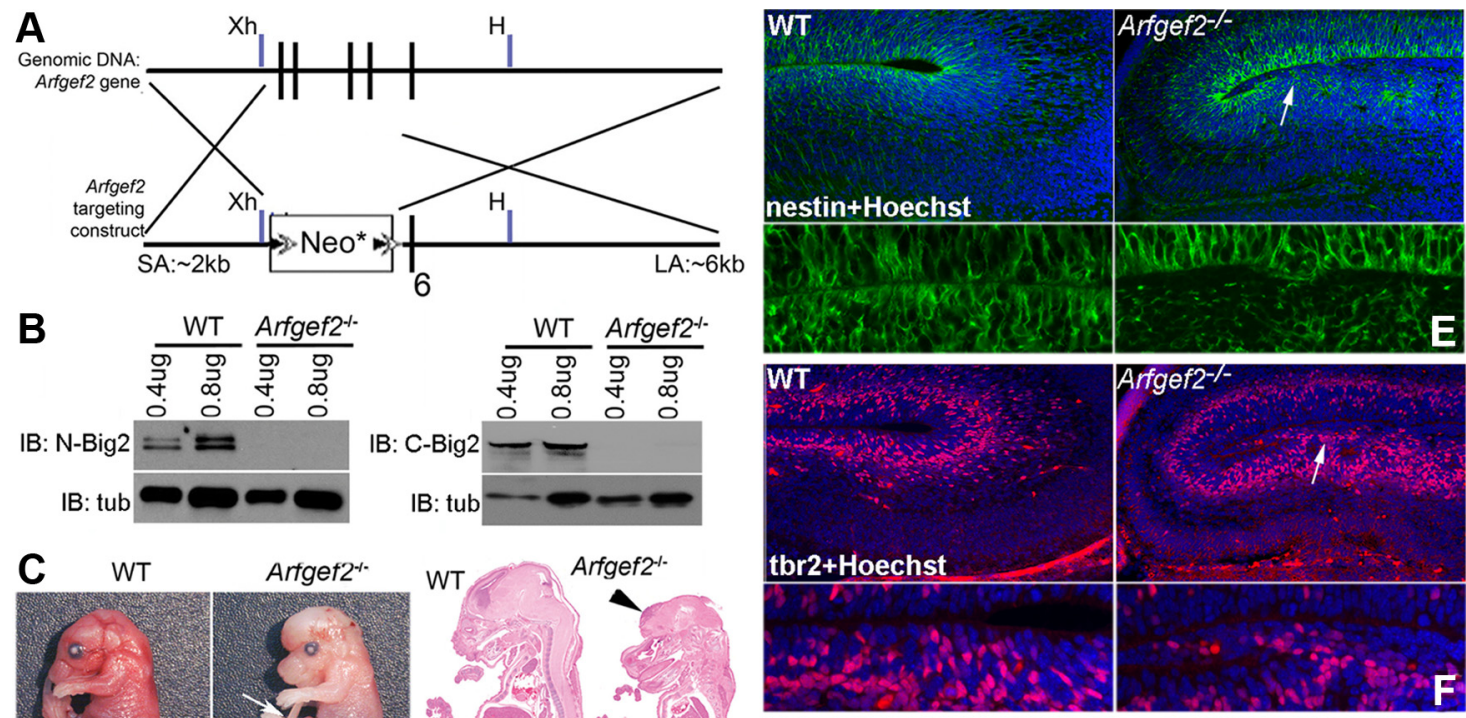

IB: tub

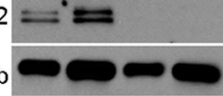

IB: C-Big2 -
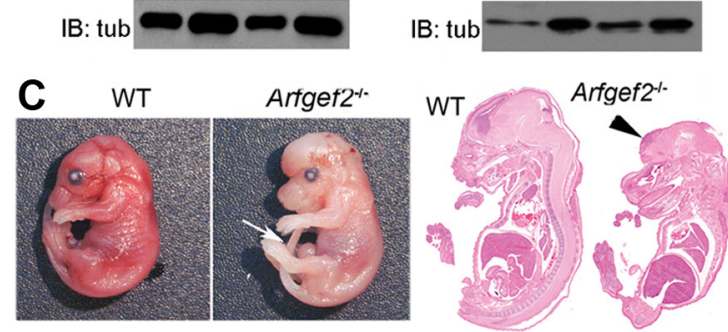

D WT

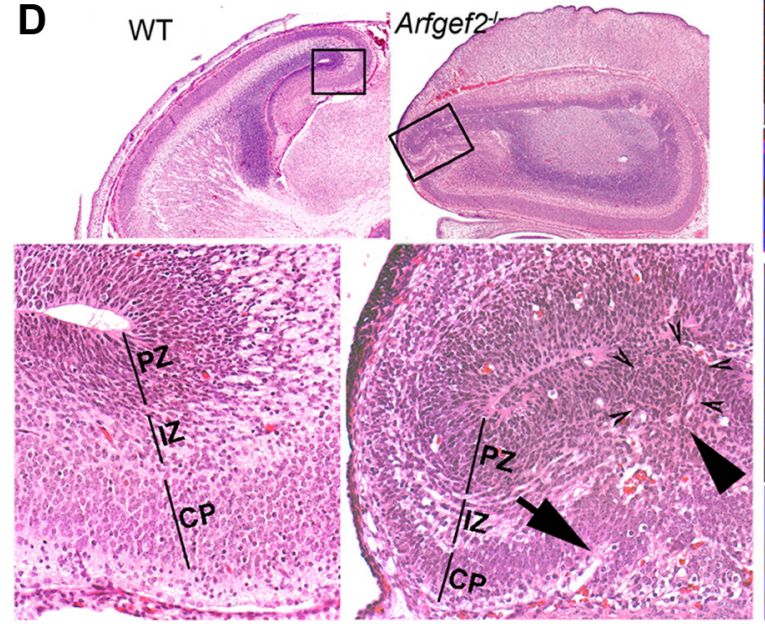

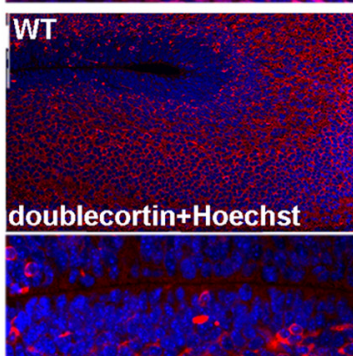
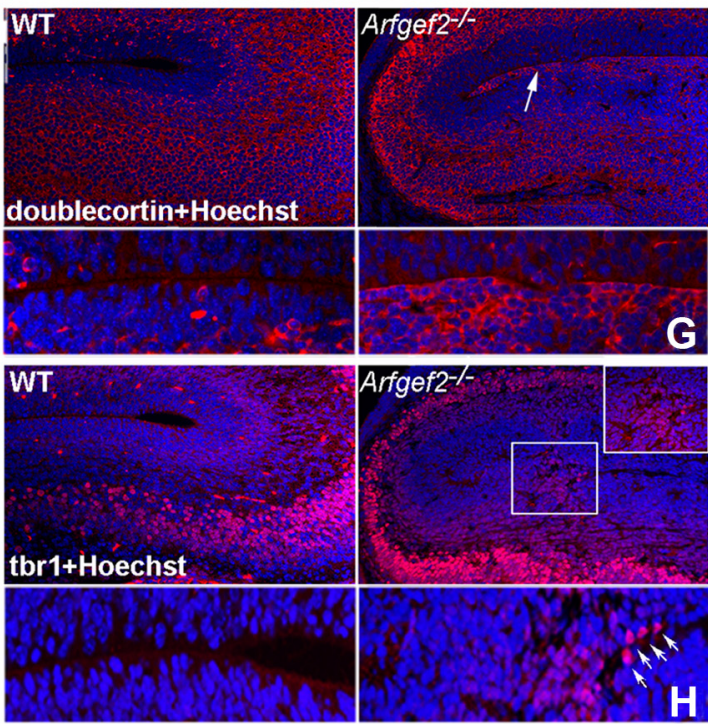

Figure 1. Arfgef2 ${ }^{-1-}$ mice develop various cortical malformations, including PH and ectopic distribution of various neural markers in the developing cortex. $A$, Targeting strategy for generating Arfgef $2^{-\prime-}$ mice. A LoxP-flanked Neo cassette was inserted downstream of a $2.0 \mathrm{~kb}$ fragment containing intron 1 (SA: short arm) and upstream of a $6.0 \mathrm{~kb}$ fragment extending from intron 5 to intron 6 (LA: long arm). The targeting vector was designed to replace exons $2-5$ of the Arfgef2 gene with a Neo cassette. Xh and $\mathrm{H}$ refer to restriction enzyme sites Xho1 and Hindlll. $\boldsymbol{B}$, Immunoblot of total brain lysates with Big2 antibodies against both $\mathrm{N}$ - and C-terminal amino acids showed the absence of Big2 expression in Arfgef2 ${ }^{-1-}$ mice. Tubulin was used as a loading control. C, Photomicrographs of whole body and H\&E-stained sections showed that late gestational (E18.5) Arfgef2 ${ }^{-/}$embryos could develop exencephaly (arrowhead) and omphalocele (arrow). D, Photomicrograph of sagittal sections of the forebrain in E18.5 WT and Arfgef2 ${ }^{-1-}$ mice. Magnified images of the cerebral cortex (shown in boxes) were revealed below. Arfgef2 ${ }^{-1-}$ mice displayed PH (arrowhead) and subependymal heterotopia (arrow). $\boldsymbol{E}$, Nestin (a marker for neural progenitors) positive immunoreactivity (arrow) revealed the presence of ectopic neural progenitor cells within the PH of mutant mice. $\boldsymbol{F}$, Ectopic localization of the basal progenitor cell marker Tbr2 (arrow) within the PH of Arfgef2 ${ }^{-1-}$ mice. G, Immature postmitotic neurons stained by doublecortin within the PH in Arfgef2 $^{-1-}$ mice (arrow). H, Tbr1-positive immunoreactivity (inset) revealed the ectopic presence of a few layer six CP neurons within the PH of Arfgef2 ${ }^{-1-}$ mice (arrows). For each marker, magnified images were shown below.

The cells were grown in serum-free medium supplemented with or without $5 \%$ FBS in the bottom of the well as a chemoattractant for $16-18 \mathrm{~h}$. The cells that traveled through the membrane were stained with $0.5 \%$ crystal violet, photographed, and quantified under light microscopy. The results were collected from four individual experiments.

\section{Results}

PH formation in Arfgef2 $2^{-/-}$mice

Although the radiographic findings of heterotopia in humans who harbor ARFGEF2 mutations implicate some disruption of neuronal migration, the investigation of BIG2 and its role in cortical development was limited without an animal model. We therefore engineered an Arfgef $2^{-1-}$ mutant mouse by replacing exons 2-5 of the Arfgef2 gene with a Neo cassette (Fig. 1A). The complete absence of Big2 expression in the Arfgef2 $2^{-/-}$mouse was verified by PCR and Western blotting. The inability to detect Big2 protein using multiple $\mathrm{N}$ - and C-terminal antibodies [amino acids surrounding leucine-1527 (Novus) and amino acids 232-241 (Sigma) (Yamaji et al., 2000)] confirmed the absence of splice variants derived from the Arfgef 2 construct design (Fig. $1 B$ ).

Big2 expression was localized within progenitors along the VZ and subventricular zone (SVZ) of the cerebral cortex at midgestation and, more specifically, the ventricular neuroependyma ( $\mathrm{Lu}$ et al., 2006; see Fig. 4). By E18.5, 12.5\% of Arfgef $2^{-\prime-}$ and $8.25 \%$ of Arfgef $2^{+/-}$mice developed exencephaly (arrowhead) and midline gut closure defects (arrow) ( $n=62$ mice; Fig. $1 C)$. Despite the severe abnormal gross morphology of the brain, a relatively normal CP was often observed in Arfgef $2^{-/-}$mice. Only Arfgef $2^{-/-}$mice with exencephaly exhibited several cerebral cortical 
malformations, including PH (arrowheads), subependymal heterotopia (arrow), and abnormal cortical involutions (Fig. $1 D$ ). Cells found within the $\mathrm{PH}$ displayed immunoreactivity to nestin (marker for neural progenitor cells; Fig. $1 E$ ), Tbr2 (marker for basal progenitor cells; Fig. $1 F$ ), doublecortin (marker for immature postmitotic neurons; Fig. $1 G$ ), and Tbrl (staining for cortical layer VI neurons; Fig. $1 H$ ). The nestin staining also suggested disruption of the radial glia in some regions with loss of integrity of the neuroependyma. Overall, these findings indicate that the loss of Big2 in exencephalic Arfgef2 $2^{-1-}$ mice causes $\mathrm{PH}$ formation associated with the abnormal positioning of various cell types along the ventricular surface. The varied composition of neurons and neuronal progenitors along the ventricular lining of Arfgerf $2^{-1-}$ embryos, as well as predominant expression of Big2 protein along the $\mathrm{VZ}$ and SVZ, suggest that Big2 has a functional role in the initiation and/or maintenance of neuronal migration and that non-cellautonomous processes, such as mechanical stressors, can contribute to heterotopia formation.

Loss of Big2 function causes defects in cell-autonomous neuronal motility Previous studies have suggested that loss of Big2 function through BFA inhibition results in denudation of the neuroependyma and heterotopia formation (Ferland et al., 2009). Although non-autonomous processes appeared to contribute to $\mathrm{PH}$ in these mice (given the apparent disruption of the radial glial scaffolding by nestin staining; Fig. 1), previous studies have also shown that loss and gain of FlnA function can impair neuronal migration (Nagano et al., 2004; Sarkisian et al., 2006). It was unclear whether cell-autonomous defects in neuronal motility could contribute in part to the mutant Arfgef 2 phenotype. Thus, we examined whether loss of Big2 function caused defects in neuronal motility by tracing the radial migration of neurons through the developing cerebral cortex. Only Arfgef $2^{-/-}$ mice without exencephaly were used in the subsequent experiments to exclude possible confounding effects from the exencephaly. We first explored neuronal motility in WT and Arfgef $2^{-/-}$mice using BrdU, a nucleotide analog incorporated into DNA during S-phase mitosis. We exposed E14.5 embryos to BrdU and determined the migratory fates of BrdU-positive cells $4 \mathrm{~d}$ later at E18.5 in regions in which the cortical width appeared intact. The loss of Big2 expression in Arfgef $2^{-1-}$ mice resulted in an increase in the percentage of BrdU-positive cells within the $\mathrm{PZ}$ at the expense of the $\mathrm{CP}$ (Fig. 2A). Given that these defects could still be attributable to both cell intrinsic and extrinsic processes from the disruption of the cortical architecture seen with the null mouse, we coelectropo-
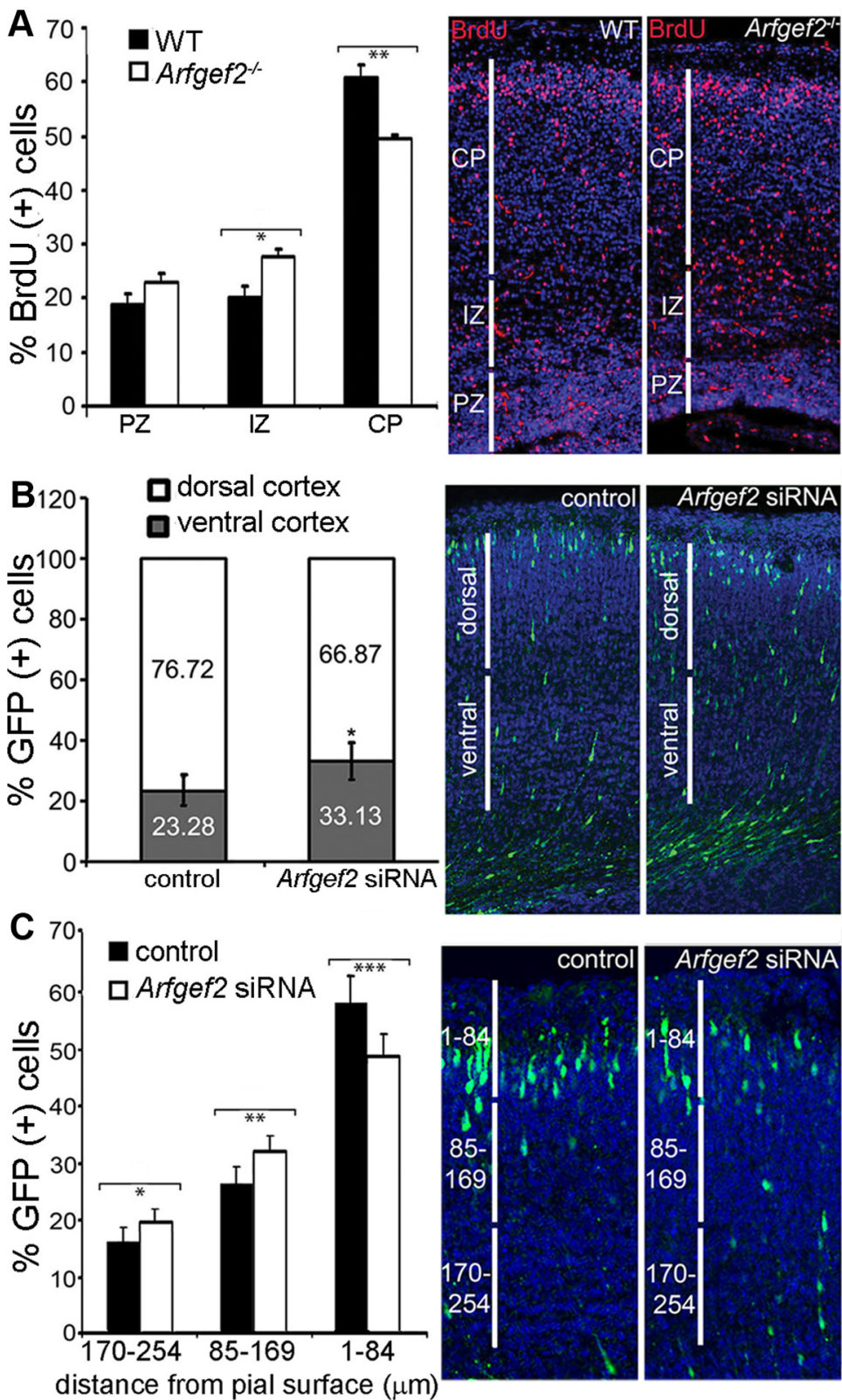

Figure 2. Loss of Big2 causes defects in neuronal migration in Arfgef2 ${ }^{-/-}$mice. $A$, The percentage of BrdU-positive cells within the IZ was significantly increased in Arfgef $2^{-1-}$ compared with WT mice $\left(n=3,{ }^{*} p<0.01\right)$. Conversely, the percentage of BrdU-positive cells within the CP was significantly decreased in Arfgef $2^{-\prime-}$ mice compared with WT mice $\left(n=3,{ }^{* *} p<0.02\right)$. Images (right) display BrdU immunoreactivity in WT and Arfgef2 ${ }^{-/-}$cortices. Pregnant E14.5 dams were injected with BrdU, and the migratory fates of these BrdU-positive neurons were examined at E18.5. B, Arfgef2 siRNA-mediated knockdown of Big2 resulted in impaired neuronal migration within the CP. The percentage of GFP-positive cells within the ventral half of the CP as opposed to the dorsal half was significantly greater in embryos electroporated with Arfgef2 siRNA compared with control siRNA $\left(n=3,{ }^{*} p<0.01\right)$. Intraventricular in utero electroporation was performed with coelectroporation of GFP with Arfgef2 siRNA or nontargeting oligonucleotides at E14.5, and mice were examined at E18.5. C, Secondary analyses of the in utero electroporation demonstrated that loss of Big2 function led to progressively fewer progenitors reaching the upper cortical layers $\left(n=3,{ }^{*} p<0.05\right.$, ${ }^{* *} p<0.01,{ }^{* * *} p<0.001$ ). Six or more serial sections were analyzed from each mouse brain.

rated GFP with either nontargeting or Arfgef2 siRNA oligonucleotides in E14.5 WT mouse embryos and tracked the migration of GFP-positive cells at E18.5. Although we found no significant difference in the total percentage of cells electroporated with Arfgef2 siRNA that were able to migrate into the cortex relative to control, siRNA-mediated knockdown of Arfgef2 led to an increased percentage of GFP-positive cells within the deeper (ventral) half as opposed to the more superficial (dorsal) half of the $\mathrm{CP}$ (Fig. 2B). Quantification of GFP-positive Arfgef2 siRNA electroporated cells, relative to 


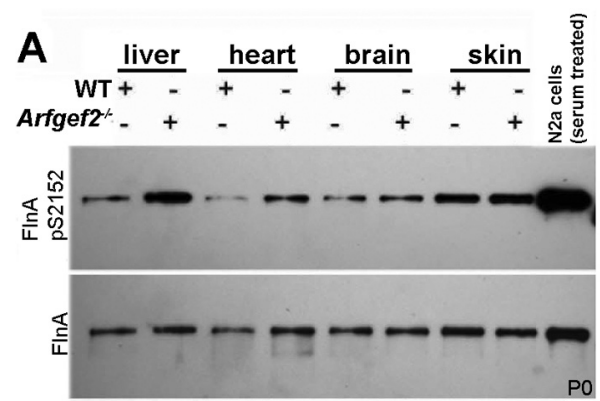

B
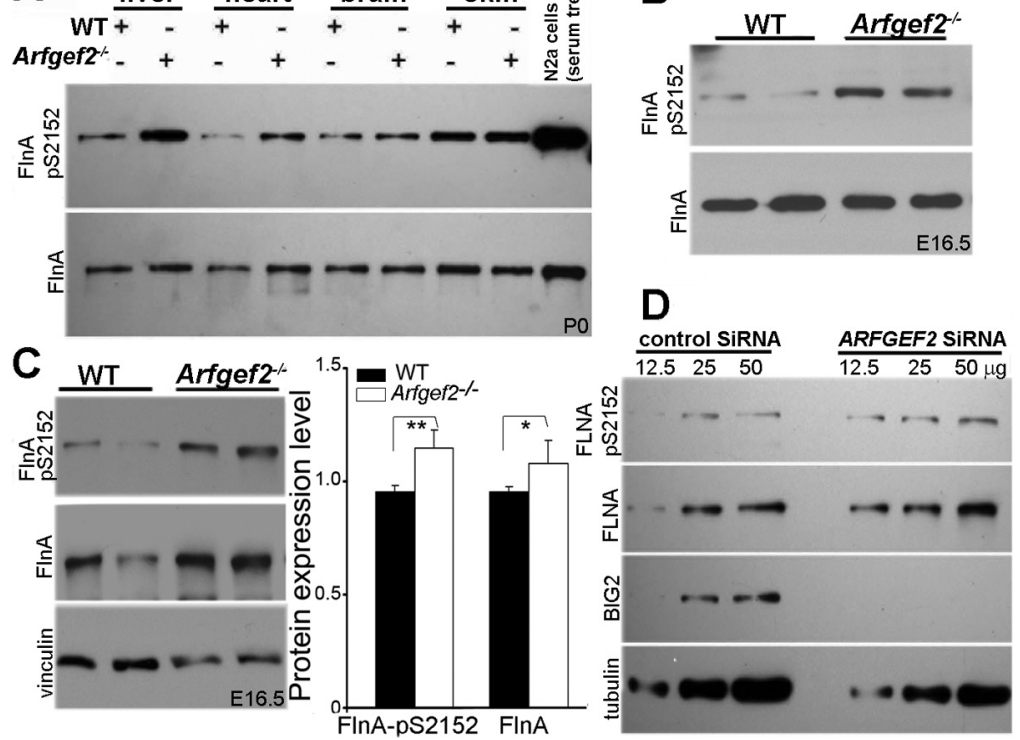

Figure 3. Loss of BIG2 enhances FInA expression and phosphorylation at Ser2152. A, Western blot displayed levels of phosphorylated FInA Ser2152 detected in liver, heart, brain, and skin tissues obtained from PO WT and Arfgef2 ${ }^{-1-}$ mice, whereas total levels of FlnA served as loading control. Serum-treated Neuro2a (N2a) cell lysates were used as a control. $\boldsymbol{B}$, Western blot showed increased level of phosphoFInA S2152 relative to normalized total FInA levels in E16.5 mouse whole-brain lysate. C, Increased expression level of both FInA and phosphoFInA Ser2152 relative to a housekeeping gene, vinculin, in forebrain tissues from E16.5 WT and Arfgef2 ${ }^{-1-}$ embryos. Quantification of FInA and phosphoFInA Ser2152 levels in E16.5 Arfgef2 ${ }^{-1}$ and WT mice was shown on the right ( $n=4$ mice per variable, ${ }^{*} p<0.5,{ }^{* *} p<0.01$ ). D, ARFGEF2 siRNA-mediated knockdown of BIG2 increased FLNA Ser2152 phosphorylation levels by Western blotting analyses. CHP100 cells were transfected with either control nontargeting siRNA or ARFGEF2 siRNA oligonucleotides, and $12.5,25$, or $50 \mu \mathrm{g}$ of cell lysate was loaded for the detection of FLNA Ser2152 phosphorylation levels. CHP100 cells transfected with ARFGEF2 siRNA oligonucleotides resulted in the loss of BIG2 expression.

their distance from the pial surface, demonstrated a similar and progressive shift toward locations in the deeper layers of the CP (Fig. $2 C)$. Together, the BrdU and electroporation findings indicate that loss of Big2 not only can alter neuronal migration through disruption along the ventricular lining but also appears to disrupt neuronal migration through cell intrinsic processes.

\section{Big2 regulates FlnA expression and phosphorylation}

Our findings and previous reports demonstrated that Big2 contains three PKA regulatory subunit binding sites, and FlnA contains several phosphorylation sites, including a PKA-dependent phosphorylation site at Ser2152 (Jay et al., 2000, 2004; Li et al., 2003; Woo et al., 2004). We verified an endogenous interaction between BIG2 and PKA-RI $\beta$ regulatory subunits and showed that catalytic PKA phosphorylates FLNA at Ser2152 in vitro (data not shown). These observations raised the possibility that Big2 might promote FlnA phosphorylation. We therefore examined phosphoFlnA Ser2152 levels in different tissues from P0 mouse by Western blotting analysis. PhosphoFlnA Ser2152 levels, however, were actually increased in the heart, liver, and brain, relative to normalized FlnA levels in each of the organ systems, after loss of Big2 function (Fig. 3A). To confirm the increased phosphoFlnA levels, we repeated the Western blotting in E16.5 Arfgef2 $2^{-/-}$ and WT mouse brain tissue. PhosphoFlnA Ser2152 was increased at both ages relative to unphosphorylated FlnA (Fig. 3B). Moreover, we demonstrated that both FlnA and phosphoFlnA Ser2152 protein levels were increased in the forebrain of E16.5 null mice by $\sim 10$ and $\sim 15 \%$, respectively, when normalized to a housekeeping gene such as vinculin (Fig. 3C). Finally, acute Big2 knockdown by transient transfection of Arfgef2 siRNA in CHP100 cells yielded the same results, albeit to a lesser degree (Fig. $3 D)$. Thus, Big2 regulates both FlnA and phosphoFlnA levels, albeit not likely through Big2-regulated, PKA-dependent phosphorylation of FlnA.

\section{FLNA and BIG2 interact and colocalize within neural progenitor cells along the ventricular surface}

Given that FlnA expression was regulated by Big2, we investigated whether these proteins physically interacted with one another. We identified an endogenous physiological interaction between full-length FLNA and BIG2 proteins by coimmunoprecipitation in CHP100 cell lysates (Fig. 4A). Next, we used a directed yeast two-hybrid assay to explore the regions of FLNA and BIG2 necessary for binding and to confirm direct, as opposed to indirect, binding. Yeast cells expressing either a BIG2-N-terminal region (BIG2-NT amino acids 1-654) or a BIG2-C-terminal region (BIG2-C1 amino acids 786-1452 or BIG2-C2 amino acids 1120-1785) in combination with the multiple receptor binding domain of FLNA (amino acids 2167-2648) had enhanced growth in negative selection media and activation of the LacZ reporter (Fig. 4B). Among these transformants, cells expressing the BIG2-NT construct had the greatest relative growth and X-gal staining. Activation of the LacZ reporter was not detected in yeast transformed with the BIG2 or FLNA constructs alone. We then verified the yeast two-hybrid findings by testing for interactions between the receptor binding domain of FLNA and either full-length or fragments of BIG2 by immunoprecipitation. Myc-tagged FLNA (amino acids 2167-2648; Fig. 4D) was detected in full-length HAtagged BIG2 immunoprecipitation. Additionally, the receptor binding domain of FLNA interacted with both GFP-tagged BIG2-NT (amino acids 1-654; Fig. 4E) and BIG2-CT (amino acids 786-1785; Fig. $4 F$ ) by coimmunoprecipitation. To further refine the region of BIG2 necessary for binding, we focused on the BIG2-NT fragment because it had the strongest interaction with FLNA by yeast twohybrid binding. Myc-FLNA was detected in GFP-tagged BIG2-NT2 (amino acids 221-654; Fig. 4H) and BIG2-NT3 (amino acids 329547; Fig. 4I) but not BIG2-NT1 (amino acids 1-441; Fig. 4G) immunoprecipitation, suggesting that amino acids $441-547$ of BIG2 are in part required for binding to FLNA. Final confirmation of a Big2-FlnA interaction was found with the observed colocalization of endogenous FlnA and Big2 along the neuroependymal ventricular surface of the brain and within neural progenitor cells in E16.5 mouse cortices (Fig. $4 \mathrm{~J}$ ). Moreover, in cultured mouse neural progenitor cells, FlnA and Big2 colocalized at the perinuclear region (arrowhead) and the cell membrane (arrow) (Fig. $4 K$ ). Overall, these data indicate that FlnA and Big2 directly interact and are colocalized along the neuroependymal surface and in neural progenitor cells.

\section{Cell migration is impaired by enhanced phosphorylation of FLNA}

Overexpression of FLNA has been reported to impair migration (Nagano et al., 2004; Sarkisian et al., 2006). To explore the role of 
A

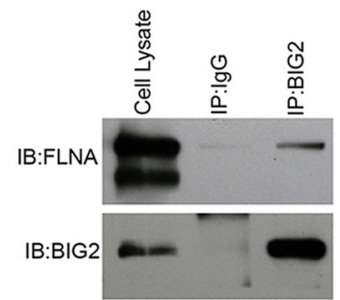

C

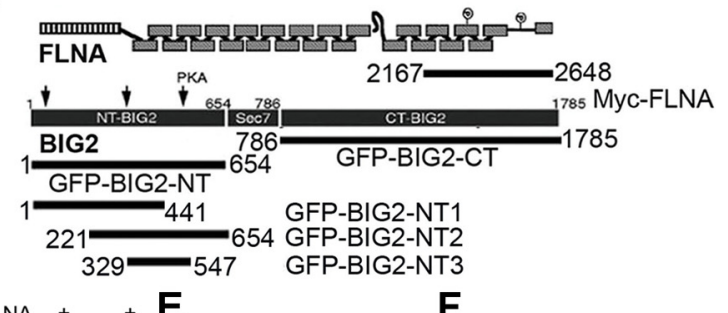

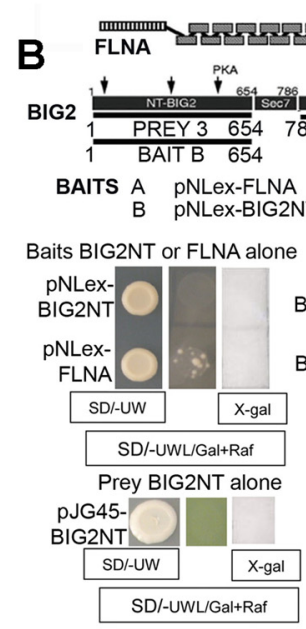

$\mathrm{J}$

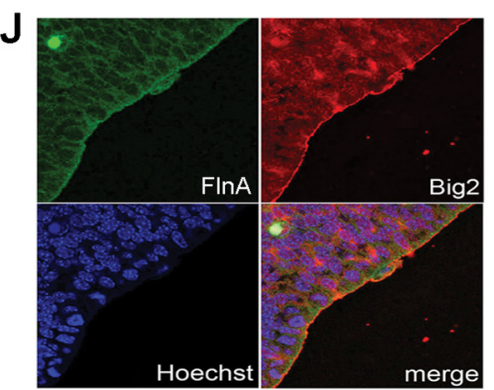

Bait-Prey

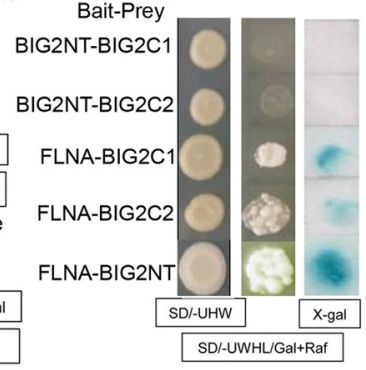

1 pJG45-BIG2C1

pJG45-BIG2C2

pJG45-BIG2NT

G

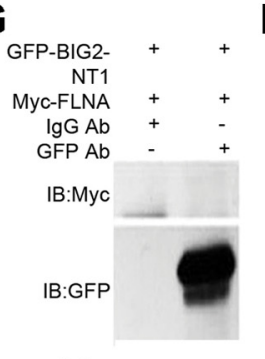

K

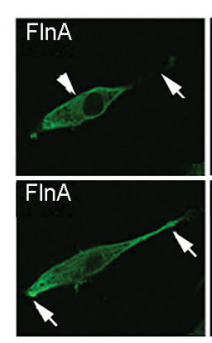

$\mathbf{F}$
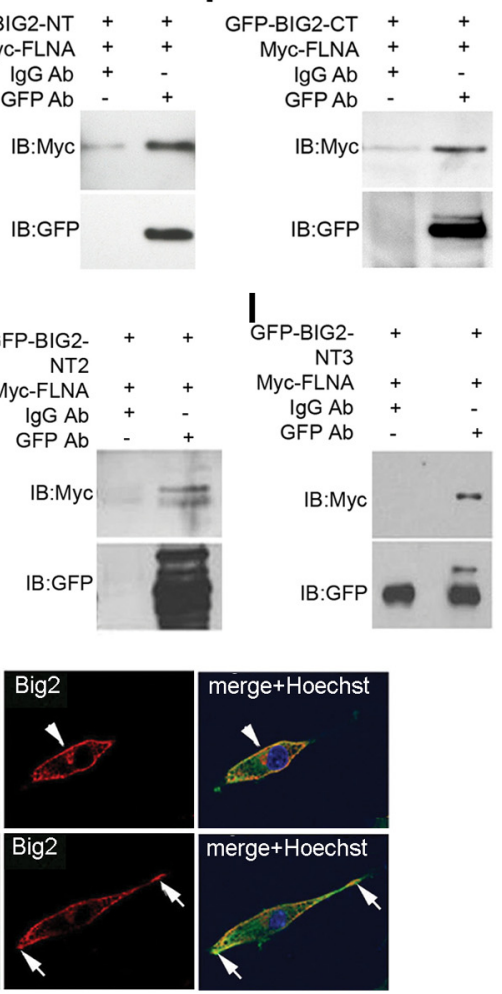

Figure 4. FLNA interacts and colocalizes with BIG2. $A$, Endogenous FLNA coimmunoprecipitated with endogenous BIG2 in human CHP100 neuroblastoma cells. $B$, The receptor binding domain of FLNA (amino acids 2167-2648) interacted with C-terminal (amino acids 786-1452 and 1120-1785) and N-terminal (amino acids 1-654) fragments of BIG2 by directed yeast two-hybrid analysis. Schematic details the regions of FLNA and BIG2 that were used for the baits and preys. Photo images displayed yeast colony growth and $\beta$-galactosidase staining. Activation of the LacZ reporter was not observed in yeast expressing BIG2-NT or FLNA (Baits) or BIG2-NT (Prey) alone (bottom left). Expression of BIG2-NT (Bait) with BIG2-C1 or BIG2-C2 (Preys) did not yield growth. Expression of FLNA (Bait) with BIG2-C1, BIG2-C2, or BIG2-NT (Preys) resulted in growth enhancement and color development (bottom right). C, Schematic detailing the extent of the Myc-FLNA and BIG2 constructs used for coimmunoprecipitation. D, Myc-FLNA (amino acids 2167-2648) was detected in HA-BIG2 (full length) immunoprecipitation. Western blot displayed the presence of Myc-FLNA in HA (right) but not lgG (left) immunoprecipitate samples. The Myc-FLNA receptor binding region could be coimmunoprecipitated by both the GFP-BIG2-NT (amino acids 1-654) (E) and GFP-BIG2-CT (amino acids 786-1785) (F) fragments. Myc-FLNA was detected in GFP-BIG2-NT2 (amino acids 221-654) (H) and GFP-BIG2-NT3 (amino acids 329-547) (I) but not in GFP-BIG2-NT1 (amino acids 1-441) (G) immunoprecipitations. This observations suggest that the FLNA receptor binding region binds BIG2 between amino acids 441 and 547 .J, Colocalization of FlnA and Big2 along the ventricular lining of the E16.5 mouse cortex. Hoechst staining delineated the nucleus and stained DNA. $K$, Confocal images displayed the colocalization of FInA and Big 2 within the perinuclear (arrowhead) and peripheral (arrows) domains of a primary neural progenitor cell. Hoechst staining delineated the nucleus and stained DNA. IB, Immunoblot; IP, immunoprecipitation.

FLNA overexpression and phosphorylation in neural migration, we used a transwell assay to evaluate the migration of CHP100 neural cells after transient transfection of either Myc vector alone or Myc-FLNA. Under similar transfection efficiency and protein expression levels, overexpression of FLNA led to $\sim 23 \%$ decrease in the number of cells traveling through the membrane (Fig. $5 A$ ). To address the potential effects of FLNA phosphorylation at Ser2152, forskolin was applied to CHP100 cells to induce activation of cAMP signaling. A $10 \mathrm{~min}$ forskolin $(10 \mu \mathrm{M})$ incubation promoted FLNA Ser2152 phosphorylation (Fig. $5 B$ ) and a significant reduction (60\%) in the migration of CHP100 cells (Fig. 5C). Because PKA activation could potentially disrupt other nonFLNA, cAMP-dependent pathways to effect migration, we generated phosphomimetic (serine to aspartic acid, S2152D) and phosphodeficient (serine to alanine, S2152A) FLNA constructs. Transient transfection of FLNA-S2152D reduced neural migration by $50 \%$ compared with FLNA-S2152A-expressing cells (Fig. $5 D)$. Finally, to address the potential effects of culture artifact, the same studies were performed in vivo after in utero electroporation of GFP alone, GFP + FLNA, GFP + FLNA-S2152A, and GFP + FLNA-S2152D constructs into the lateral ventricle of E15.5 mouse embryos. Seventy-two hours later, these E18.5 embryos were killed, and the GFP-positive cell distribution within the CP, $\mathrm{IZ}$, and PZ were analyzed. Progenitors in which phosphomimetic FlnA (S2152D) was overexpressed had fewer number of cells migrating into the $\mathrm{CP}$ by E18.5 compared with those progenitors electroporated with the phosphodeficient control FlnA (S2152A; Fig. $5 E, F)$. Collectively, these findings suggest that phosphoryla- 


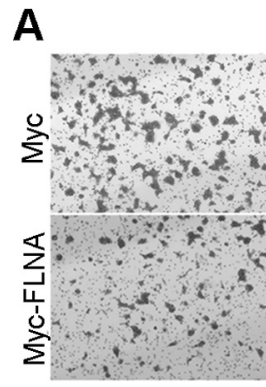

$\mathbf{E}$

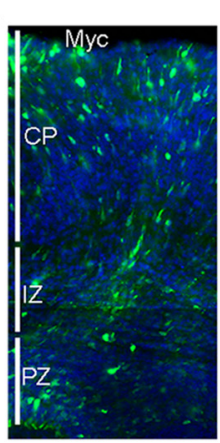

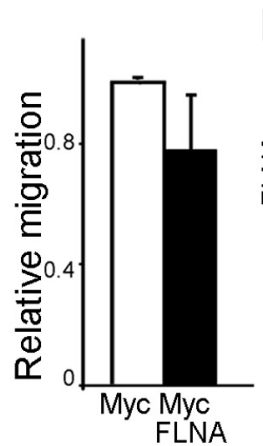

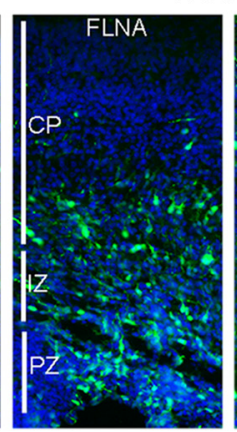

B

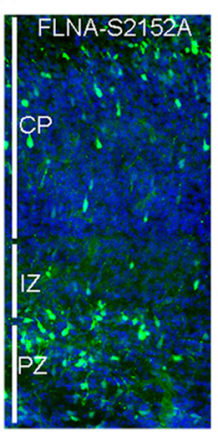

starve forskolin

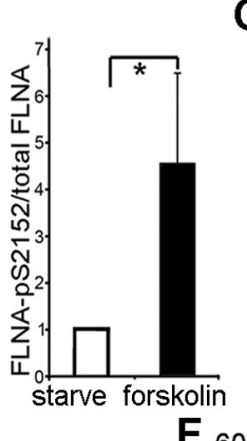

C

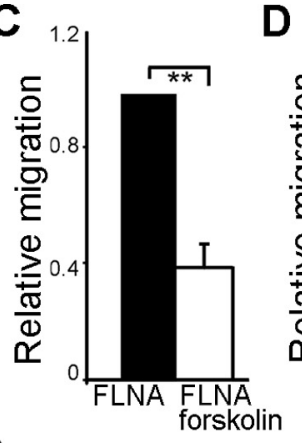

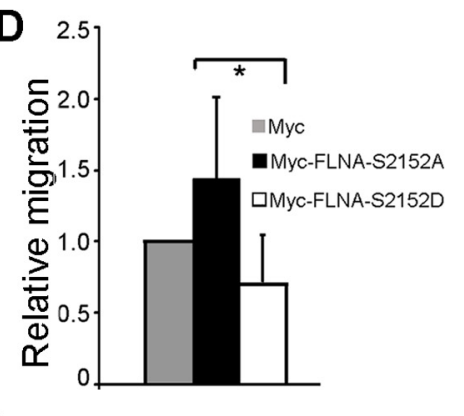
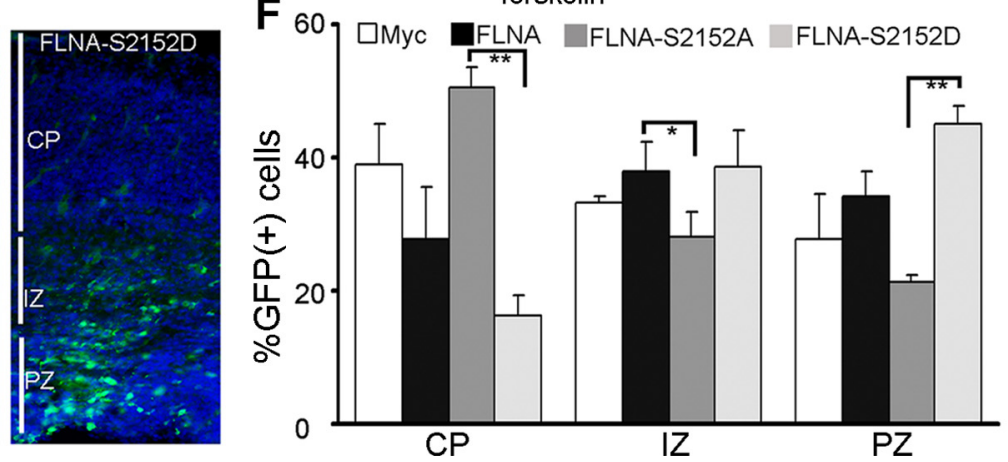

Figure 5. Increased phosphorylation of FLNA at Ser2152 impairs cell migration. A, Bright-field photomicrographs showed that the cells transfected with either Myc or Myc-FLNA migrated to the lower surface of the transwell membrane. The cells were stained with $0.5 \%$ crystal violet for visualization. Quantification of the number of Myc-FLNA-positive cells that had migrated in transwell assay relative to Myc-positive cells alone was shown to the right $(n=3)$. $\boldsymbol{B}$, Incubation of serum-starved CHP100 cells with forskolin $(10 \mu \mathrm{m})$ for $10 \mathrm{~min}$ led to an $\sim 3.5$-fold increase in the ratio of phosphorylated FLNA Ser2152/total FLNA $\left(n=3,{ }^{*} p<0.05\right)$. C, Forskolin treatment significantly reduced the migrated CHP100 cell number to $60 \%$ compared with the control group in the transwell assay $\left(n=4,{ }^{* *} p<0.01\right)$. D, Bar graph showed reduced migrating cell numbers in the Myc-FLNA-S2152D (serine 2152 to aspartic acid) group compared with the Myc-FLNA-S2152A (serine 2152 to alanine) group $\left(n=4,{ }^{*} p<0.05\right)$. All transwell assays were calculated from three or four triplicate individual experiments. $\boldsymbol{E}$, Representative fluorescent photomicrographs showed GFP-positive cells after in utero electroporation of GFP alone or GFP with FLNA, FLNA-S2152A, and FLNA-S2152D. Hoechst staining was used to define the CP layers. More GFP progenitors were observed near the ventricular lining in mice electroporated with the FLNA-S2152D construct. $\boldsymbol{F}$, Bar graphs showed the percentage of GFP-positive cells in the CP, IZ, and PZ compared with total GFP cell number $72 \mathrm{~h}$ after in utero electroporation ( $n=4$ per experimental variable; data represents the mean $\pm \mathrm{SE} ;{ }^{*} p<0.5$, ${ }^{* *} p<0.01$ ).

tion of FLNA at Ser2152, more so than FlnA alone, is responsible for the impairment in neuronal migration.

\section{PhosphoFLNA redistributes FLNA localization and alters actin-focal adhesion organization}

Cell migration involves continuous actin cytoskeletal remodeling and focal adhesive complex changes. Phosphorylation causes FLNA cellular relocalization along the cell membrane and is required for crosslinking of actin fibers, thereby playing an important role in cell cytoskeletal dynamics (Vadlamudi et al., 2002; Woo et al., 2004). To understand how phosphoFLNA Ser2152 might affect these processes, we transfected Myc-FLNA-S2152A and Myc-FLNA-S2152D constructs into CHP100 cells and examined the cellular distribution and colocalization of FLNA with actin fibers. The cells expressing FLNA-S2152A appeared concentrated and colocalized with actin fibers along the membrane ruffles. In contrast, Myc-FLNA-S2152D staining was seen diffusely within the cell cytoplasm (Fig. 6A,B). We did not see significant actin fiber changes between these two transfection conditions. To avoid potential artifact from endogenous FLNA in CHP100 cells, we reexpressed the plasmids in M2 cells (FLNA depleted melanoma cells; Fig. 6C). Similar to the result in CHP100 cells, the FLNA-S2152A clearly localized around the cell surface and overlapped with actin fiber expression, whereas the FLNA-S2152D was also seen extensively in the cytoplasm. Actin fibers that polymerize along the leading edge of migratory cells direct local protrusions through a series of protein complex assembly and disassembly events, involving FLNA. We therefore asked whether phosphorylation at Ser2152 changes the binding affinity of FLNA to actin fibers as suggested in the fluorescence images. We extracted Triton X-100 insoluble actin fibers from CHP100 cells overexpressing FLNA-S2152A and FLNAS2152D. After Western blotting, we observed increased FLNAS2152A in the Triton X-100 insoluble fraction compared with FLNA-S2152D (Fig. 6D,E). These observations suggest that FlnA phosphorylation state at Ser2152 alters the FlnA-actin binding affinities.

FlnA is often thought to serve as a scaffolding protein and changes in FlnA-actin affinity could alter the stability of various proteins. Because FLNA couples the cell cytoskeleton to extracellular matrix and regulates focal adhesion turnover (Xu et al., 2010), we examined whether phosphorylation of FLNA Ser 2152 affects focal adhesion structure and expression levels. No focal adhesion protein level changes were appreciated in FAK, phospho-FAK397, paxillin, phospho-paxillin118, and vinculin between phosphomimetic and phosphodeficient group in CHP100 cell lysates after Western blotting analyses (data not shown). However, FlnA phosphorylation (FLNA-S2152D) led to fewer but larger paxillin-staining focal adhesions. Conversely, MEF cells transfected with FLNA-S2152A caused an increase in the number but smaller size of paxillin staining focal adhesions (Fig. $6 F, G$ ). The vinculin staining did not show significant changes between these two groups (data not shown). These results indicate that elevated phosphorylation of FLNA Ser2152 causes FLNA subcellular redistribution and alters actin binding affinity, which may, in turn, lead to changes in focal adhesion number and size. This change in focal adhesion stability would directly correlate to the impairments in neuronal migration. 

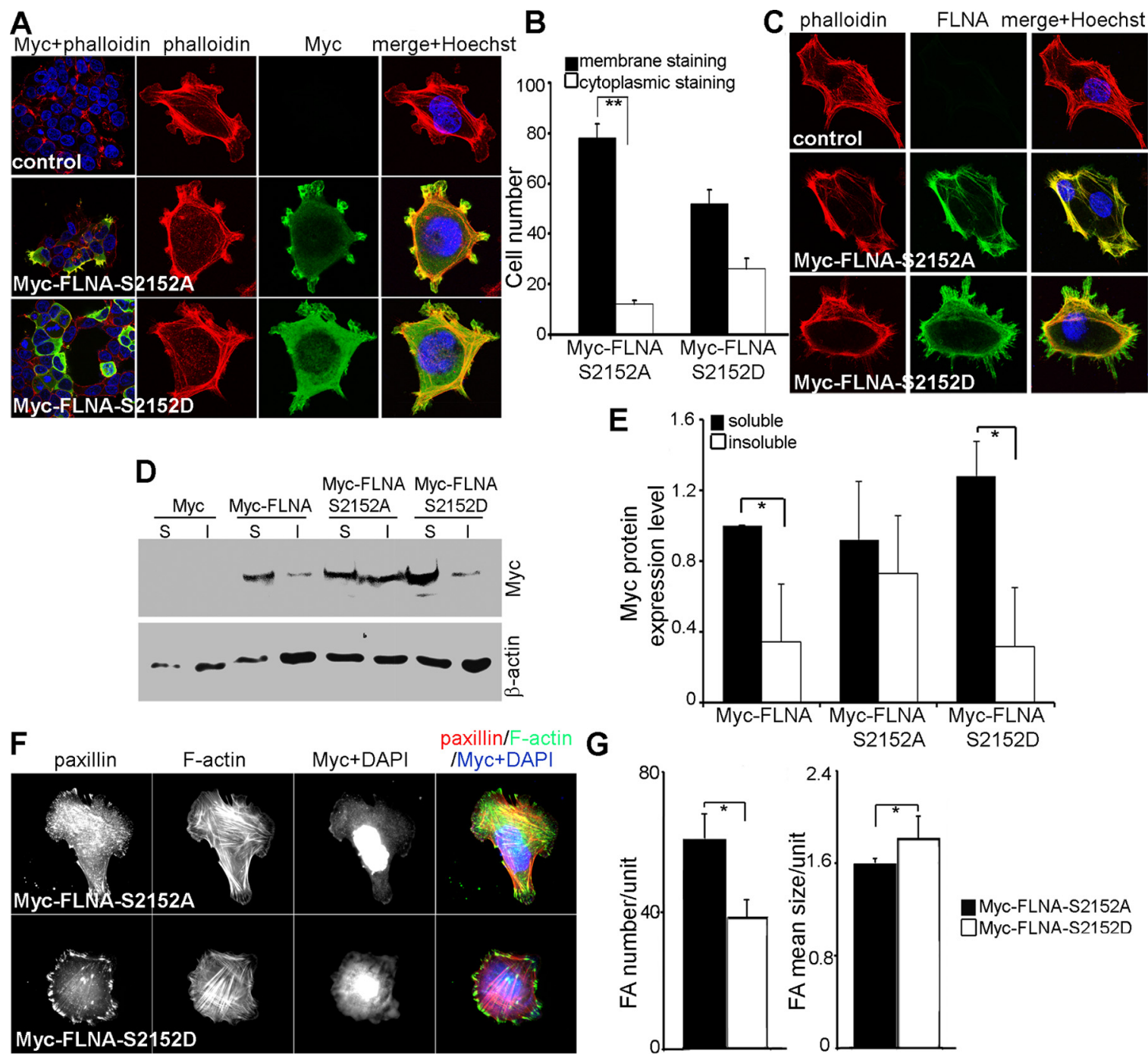

Figure 6. Phosphorylation of FLNA at Ser2152 alters FLNA subcellular distribution, its binding affinity to actin fibers, and focal adhesion structure. A, CHP100 cells transfected with Myc-FLNAS2152A or FLNA-S2152D were stained with Myc antibody and phalloidin. Myc-FLNA-S2152A accumulated in cell protrusions and colocalized with actin fibers, whereas FLNA-S2152D was found to be diffusely localized throughout the cytoplasm. A low-magnification field of transfected cells with altered cellular distribution was displayed in the left column. $\boldsymbol{B}$, Statistical analyses of subcellular distribution of Myc-FLNA-S2152A and FLNA-S2152D quantified from a total of $80-100$ single cells from three independent experiments $\left({ }^{* *} p<0.01\right)$. C, Similar to the CHP100 studies, FLNA immunostaining of Myc-FLNA-S2152A and FLNA-S2152D in transfected M2 cells (FLNA-depleted melanoma cells). The Myc-FLNA-S2152A appeared colocalized with actin fibers near the cell surface, and FLNA-S2152D was found to be distributed throughout the cytoplasm. D, Western blot analyses demonstrated increased binding affinity of FLNA-S2152A to actin fibers in Triton X-100 insoluble fractions compared with FLNA-S2152D transfected cells. $\boldsymbol{E}$, Quantification of Western blot experiments in $D\left(n=3,{ }^{*} p<0.5\right)$. $\boldsymbol{F}$, Paxillin staining showed increased numbers but reduced size of focal adhesion in Myc-FLNA-S2152A-expressing MEF cells. Myc-FLNA-S2152D-expressing MEFs displayed fewer but larger paxillin-associated focal adhesion. G, Statistical analyses of paxillin staining for focal adhesions in Myc-FLNA-S2152A and Myc-FLNA-S2152D MEFs. The focal adhesion number was quantified relative to total cell surface area using NIH Image software. The focal adhesion size was generated from quantifying the area of cellular fluorescent labeling divided by the number of focal adhesions in the cell ( $n=3$ independent experiments, 15-20 cells per variable). FA, Focal adhesion $\left({ }^{*} p<0.05\right)$.

\section{Discussion}

$\mathrm{PH}$ is a congenital brain disorder thought to involve disruption of initial neuronal migration (Lu and Sheen, 2005). The current work suggests that two genes, FLNA and ARFGEF2, causal for this disorder interact to disrupt neural migration and the integrity of the neuroependyma. Big2 regulates the phosphorylation state of FlnA, and overexpression of phosphoFlnA impairs neural migration into the cortex. FlnA phosphorylation state dictates actin binding affinity and alters the focal adhesion sites, thereby providing a potential mechanism for regulation of neural migration and $\mathrm{PH}$ formation.

The overlapping defects in humans and mice harboring FLNA and ARFGEF2 gene mutations indicate some shared molecular function across various organ systems. Humans harboring ARFGEF2 mutations show microcephaly and $\mathrm{PH}$ (Sheen et al., 2004), and the Arfgef2 $2^{-1-}$ mouse has variable findings of $\mathrm{PH}$ as well as exencephaly and omphalocele formation. A previous study reported early embryonic lethality of a gene trap mouse with disruption of the Arfgef2 gene (Grzmil et al., 2010). In an analogous manner, human FLNA mutations lead to milder microcephaly and $\mathrm{PH}$, as well as midline cleft palates, sternal clefts, omphaloceles, and encephaloceles (Robertson et al., 2003; Robertson, 2005; Gérard-Blanluet et al., 2006; Solé et al., 2009). The null FlnA mice are embryonic lethal and therefore do not develop $\mathrm{PH}$, but they do show a disrupted neuroependymal lining, microcephaly, and midline defects, including omphalocele formation (Lian et al., 2012). Together, these findings begin to implicate FLNA and ARFGEF2 
genes in a shared molecular function regulating progenitor development.

BIG2 appears to regulate various modalities involved in neuronal migration. Our previous studies have shown that inhibition of BIG2 through BFA leads to disruption of the neuroependymal lining and heterotopia formation (Ferland et al., 2009). In the current work, Arfgef $2^{-1-}$ mice show the same disruption of the nestin-positive radial glial scaffolding and of the positioning of various neuronal progenitors directly within the VZ. In this context, disruption of glial-guided migration from loss of neuroependymal integrity and consequent disruption of radial glia might be sufficient to cause PH. Exposure of E14.5 Arfgef2 ${ }^{-/-}$ mice to BrdU and analyses at E18.5 revealed more cells within the IZ, suggesting a disruption in the intermediate stages of migration. These observations, however, would suggest that Big2 plays an additional role in intrinsic cell migration or motility. Thus, $\mathrm{PH}$ likely arises from a contribution of both cell intrinsic (neural migration) and cell extrinsic (disruption of the neuroependyma) processes.

The mechanism by which loss of Big2 function leads to increased FlnA and phosphoFlnA expression is unclear. It is unlikely that the A-kinase anchor protein sites from Big2 directly assist in FlnA phosphorylation, given that loss of Big2 function leads to upregulation and not downregulation of FlnA phosphorylation. Both Big2 and FlnA, however, have been implicated in vesicle trafficking of receptors along the cell membrane, suggesting that they regulate the stability and clearance of various proteins along the cell surface (Liu et al., 1997; Lin et al., 2001; Charych et al., 2004; Shen et al., 2006; Ravid et al., 2008). To address whether loss of Big2 impaired protein degradation or enhanced protein synthesis, we quantified the clearance of FlnA and $\beta$-integrin protein levels in Arfgef $2^{-/-}$progenitor cells at different time points after exposure to cycloheximide, an inhibitor of protein synthesis. Both proteins showed a progressive decline in expression by Western blotting after 2 hours in WT precursors. However, we observed a trend toward increased rates of clearance for FlnA in the WT as opposed to Arfgef $2^{-/}$cells, but this was not statistically significant (data not shown). The rate of degradation of $\beta$-integrin was significantly slower in the null Arfgef 2 cells, suggesting that Big2 did regulate the stability of certain receptors near the cell surface. The greater stability of the actin binding FlnA might require a longer timeframe for clearance than could be performed in the current studies to observe a significant change (as a result of cyclohexamide toxicity effects). Alternatively, phosphorylation of FlnA at Ser2152 has been shown to direct various proteins toward the cell membrane (Vadlamudi et al., 2002), and we had also previously observed that dominant-negative inhibition of Big2 impaired the delivery of FlnA to the cell membrane (Lu et al., 2006). Thus, the upregulation of phosphoFlnA might serve as a compensatory response by cells to promote FlnA function at the cell membrane. Additional studies will be necessary to address these possibilities.

The neuropathological abnormalities observed in our current work are remarkably similar to those seen in the null Mekk4 mouse (Sarkisian et al., 2006), suggesting a shared common endpoint. Mekk4 suppression leads to heterotopia formation in mice, disruption of the neuroependyma, and is associated with an increase in both FlnA and phosphoFlnA levels. MEKK4 is a mitogen-activated protein kinase kinase kinase (MAP3K) that affects the activity of downstream MAP2Ks and MAPKs, including JNK and p38 (Gerwins et al., 1997). Integrin mediates the phosphorylation of JNK and directs cell spreading and adhesion, and this can be abolished by BFA (Nguyen et al., 2000). We also observed an increase in integrin expression with loss of Big2 (data not shown). Taken collectively, FlnA interactions with both Big2 and surface receptors such as integrins suggest that it serves to integrate the signaling and activation of the JNK pathway, in part through Mekk4.

FlnA phosphorylation state may serve as a key regulator in neural migration. Several studies have demonstrated that both increased and decreased levels of FlnA phosphorylation (Ser2152) impair migration. Growth factor-mediated phosphorylation of FlnA (Ser2152) leads to increased migration of melanoma cells (Woo et al., 2004; Ravid et al., 2008). Conversely, mice deficient in Mekk4 showed both increase in FlnA and phosphoFlnA expression and impaired neuronal migration (Sarkisian et al., 2006). Based on these observations and previous reports that phosphoFlnA impaired calpain-dependent degradation of FlnA (Sarkisian et al., 2006), Sarkisian et al. raised the possibility that phosphoFlnA prevented FlnA degradation and led to increased FlnA levels, thereby inhibiting migration. Our current work, however, suggests that the phosphorylation state of FlnA may play a more pivotal role in impairing migration. In utero electroporation of phosphomimetic FlnA-S2152D impairs migration, whereas the phosphodeficient FLNA-S2152A enhances migration. Moreover, consistent with previous reports that FlnA phosphorylation reduces the binding of FlnA to actin filaments in general (Ohta and Hartwig, 1995), we find that phosphorylated FlnA (Ser2152) does not bind actin as strongly and leads to redistribution of FlnA to the cytoplasm. Overall, a balance in FlnA phosphorylation at Ser2152 may be pivotal in controlling migration. For example, increased FlnA phosphorylation is thought to impair actin binding and consequent formation and/or turnover of actin networks, which would presumably disrupt migration (Ohta and Hartwig, 1995). Conversely, phosphorylation also prevents FlnA degradation by calpain cleavage, and increased FlnA levels should promote migration by enhancing actin network formation/turnover (García et al., 2006; O'Connell et al., 2009).

Focal adhesions serve as the mechanical linkages between the extracellular matrix and intracellular actin cytoskeleton, thereby directing signaling proteins at sites of integrin (and filamin) binding and clustering. Their assembly at the leading edge and disassembly at the rear of cells are essential for migration (Vicente-Manzanares et al., 2009). Previous studies have suggested that increased cell motility is associated with smaller but more numerous focal adhesions (Xu et al., 1998; Ziegler et al., 2006). We similarly observed an increase in neural migration after overexpression of the FLNA-S2152A construct, which is associated with smaller but more numerous paxillin-associated focal adhesions. Conversely, larger and fewer paxillin-associated focal adhesions were seen in phosphomimetic FLNA-S2152D transfected cells and led to a reduction in migration. It remains unclear whether and how the phosphorylation-dependent FlnAactin binding affinity regulates focal adhesion sites. One possibility is that the increased binding affinity (FlnA unphosphorylated state) is more permissive to signal transduction and allows for FlnA and actin-dependent, dynamic changes, which may facilitate paxillin-containing focal adhesion remodeling. Similarly, the phosphomimetic mutant weakens FlnA and actin interaction, which may in turn limit actin cytoskeletal conformational or structural changes and impair focal adhesion disassembly required for migration. Nevertheless, both alterations in actin binding and increased size of paxillin focal adhesion sites could be detrimental to neural migration.

The current study uncovers an important functional link between the actin-binding FlnA and vesicle trafficking related Big2 
proteins in guiding neural migration, presumably through Big2dependent regulation of FlnA phosphorylation and association with actin-focal adhesion organization. It is important to recognize that FlnA-dependent regulation of the actin cytoskeleton will not only influence the cell shape and movement but may also govern other actin-dependent processes, namely vesicle trafficking. It will be of interest to address whether FlnA regulates expression and function of vesicle-associated proteins such as Big2 and whether disruption of this pathway also alters paxillin or other focal adhesion complexes. In this context, interactions between proteins that regulate vesicle formation (i.e., GEFs) and actin stability/turnover (i.e., filamins) may begin to provide a means by which changes in intracellular vesicular trafficking can give rise to the various neurological phenotypes associated with $\mathrm{PH}$, including disruption of the neuroependymal lining, reduction in brain size, and impairment in neuronal migration.

\section{References}

Achstetter T, Franzusoff A, Field C, Schekman R (1988) SEC7 encodes an unusual, high molecular weight protein required for membrane traffic from the yeast Golgi apparatus. J Biol Chem 263:11711-11717.

Charych EI, Yu W, Miralles CP, Serwanski DR, Li X, Rubio M, De Blas AL (2004) The brefeldin A-inhibited GDP/GTP exchange factor 2, a protein involved in vesicular trafficking, interacts with the beta subunits of the GABA receptors. J Neurochem 90:173-189.

D'Souza-Schorey C, Chavrier P (2006) ARF proteins: roles in membrane traffic and beyond. Nat Rev Mol Cell Biol 7:347-358.

Faux CH, Parnavelas JG (2007) The role of intracellular calcium and RhoA in neuronal migration. Sci STKE 2007:pe62.

Feng Y, Chen MH, Moskowitz IP, Mendonza AM, Vidali L, Nakamura F, Kwiatkowski DJ, Walsh CA (2006) Filamin A (FLNA) is required for cell-cell contact in vascular development and cardiac morphogenesis. Proc Natl Acad Sci U S A 103:19836-19841.

Ferland RJ, Batiz LF, Neal J, Lian G, Bundock E, Lu J, Hsiao YC, Diamond R, Mei D, Banham AH, Brown PJ, Vanderburg CR, Joseph J, Hecht JL, Folkerth R, Guerrini R, Walsh CA, Rodriguez EM, Sheen VL (2009) Disruption of neural progenitors along the ventricular and subventricular zones in periventricular heterotopia. Hum Mol Genet 18:497-516.

Ferland RJ, Gaitanis JN, Apse K, Tantravahi U, Walsh CA, Sheen VL (2006) Periventricular nodular heterotopia and Williams syndrome. Am J Med Genet A 140:1305-1311.

Fox JW, Walsh CA (1999) Periventricular heterotopia and the genetics of neuronal migration in the cerebral cortex. Am J Hum Genet 65:19-24.

García E, Stracher A, Jay D (2006) Calcineurin dephosphorylates the C-terminal region of filamin in an important regulatory site: a possible mechanism for filamin mobilization and cell signaling. Arch Biochem Biophys 446:140-150.

Gérard-Blanluet M, Sheen V, Machinis K, Neal J, Apse K, Danan C, Sinico M, Brugières P, Mage K, Ratsimbazafy L, Elbez A, Janaud JC, Amselem S, Walsh C, Encha-Razavi F (2006) Bilateral periventricular heterotopias in an X-linked dominant transmission in a family with two affected males. Am J Med Genet A 140:1041-1046.

Gerwins P, Blank JL, Johnson GL (1997) Cloning of a novel mitogenactivated protein kinase kinase kinase, MEKK4, that selectively regulates the c-Jun amino terminal kinase pathway. J Biol Chem 272:8288-8295.

Grzmil P, Enkhbaatar Z, Gundsambuu B, Oidovsambuu O, Yalcin S, Wolf S, Engel W, Neesen J (2010) Early embryonic lethality in gene trap mice with disruption of the Arfgef2 gene. Int J Dev Biol 54:1259-1266.

He M, Zhang ZH, Guan CB, Xia D, Yuan XB (2010) Leading tip drives soma translocation via forward F-actin flow during neuronal migration. J Neurosci 30:10885-10898.

Jay D, García EJ, Lara JE, Medina MA, de la Luz Ibarra M (2000) Determination of a cAMP-dependent protein kinase phosphorylation site in the $\mathrm{C}$-terminal region of human endothelial actin-binding protein. Arch Biochem Biophys 377:80-84.

Jay D, García EJ, de la Luz Ibarra M (2004) In situ determination of a PKA phosphorylation site in the C-terminal region of filamin. Mol Cell Biochem 260:49-53.

Jones HD, Moss J, Vaughan M (2005) BIG1 and BIG2, brefeldin A-inhibited guanine nucleotide-exchange factors for ADP-ribosylation factors. Methods Enzymol 404:174-184.

Jones KJ, Korb E, Kundel MA, Kochanek AR, Kabraji S, McEvoy M, Shin CY, Wells DG (2008) CPEB1 regulates beta-catenin mRNA translation and cell migration in astrocytes. Glia 56:1401-1413.

Letinic K, Sebastian R, Toomre D, Rakic P (2009) Exocyst is involved in polarized cell migration and cerebral cortical development. Proc Natl Acad Sci U S A 106:11342-11347.

Li H, Adamik R, Pacheco-Rodriguez G, Moss J, Vaughan M (2003) Protein kinase $\mathrm{A}$-anchoring (AKAP) domains in brefeldin A-inhibited guanine nucleotide-exchange protein 2 (BIG2). Proc Natl Acad Sci U S A 100:1627-1632.

Lian G, Lu J, Hu J, Zhang J, Ferland R, Sheen V (2012) Filamin A regulates neural progenitor proliferation and cortical size through Wee1dependent Cdk1 phosphorylation. J Neurosci. In press.

Lin R, Karpa K, Kabbani N, Goldman-Rakic P, Levenson R (2001) Dopamine D2 and D3 receptors are linked to the actin cytoskeleton via interaction with filamin A. Proc Natl Acad Sci U S A 98:5258-5263.

Liu G, Thomas L, Warren RA, Enns CA, Cunningham CC, Hartwig JH, Thomas G (1997) Cytoskeletal protein ABP-280 directs the intracellular trafficking of furin and modulates proprotein processing in the endocytic pathway. J Cell Biol 139:1719-1733.

Lu J, Sheen V (2005) Periventricular heterotopia. Epilepsy Behav 7:143-149.

Lu J, Tiao G, Folkerth R, Hecht J, Walsh C, Sheen V (2006) Overlapping expression of ARFGEF2 and Filamin A in the neuroependymal lining of the lateral ventricles: insights into the cause of periventricular heterotopia. J Comp Neurol 494:476-484.

Nadarajah B, Parnavelas JG (2002) Modes of neuronal migration in the developing cerebral cortex. Nat Rev Neurosci 3:423-432.

Nagano T, Morikubo S, Sato M (2004) Filamin A and FILIP (Filamin A-Interacting Protein) regulate cell polarity and motility in neocortical subventricular and intermediate zones during radial migration. J Neurosci 24:9648-9657.

Nguyen BP, Gil SG, Carter WG (2000) Deposition of laminin 5 by keratinocytes regulates integrin adhesion and signaling. J Biol Chem 275:31896-31907.

O'Connell MP, Fiori JL, Baugher KM, Indig FE, French AD, Camilli TC, Frank BP, Earley R, Hoek KS, Hasskamp JH, Elias EG, Taub DD, Bernier M, Weeraratna AT (2009) Wnt5A activates the calpain-mediated cleavage of filamin A. J Invest Dermatol 129:1782-1789.

Ohta Y, Hartwig JH (1995) Actin filament cross-linking by chicken gizzard filamin is regulated by phosphorylation in vitro. Biochemistry 34:6745-6754.

Ravid D, Chuderland D, Landsman L, Lavie Y, Reich R, Liscovitch M (2008) Filamin A is a novel caveolin-1-dependent target in IGF-I-stimulated cancer cell migration. Exp Cell Res 314:2762-2773.

Robertson SP (2005) Filamin A: phenotypic diversity. Curr Opin Genet Dev 15:301-307.

Robertson SP, Twigg SR, Sutherland-Smith AJ, Biancalana V, Gorlin RJ, Horn D, Kenwrick SJ, Kim CA, Morava E, Newbury-Ecob R, Orstavik KH, Quarrell OW, Schwartz CE, Shears DJ, Suri M, Kendrick-Jones J, Wilkie AO; OPD-Spectrum Disorders Clinical Collaborative Group (2003) Localized mutations in the gene encoding the cytoskeletal protein filamin A cause diverse malformations in humans. Nat Genet 33:487-491.

Sarkisian MR, Bartley CM, Chi H, Nakamura F, Hashimoto-Torii K, Torii M, Flavell RA, Rakic P (2006) MEKK4 signaling regulates filamin expression and neuronal migration. Neuron 52:789-801.

Sheen VL, Feng Y, Graham D, Takafuta T, Shapiro SS, Walsh CA (2002) Filamin A and Filamin B are co-expressed within neurons during periods of neuronal migration and can physically interact. Hum Mol Genet 11:2845-2854.

Sheen VL, Ganesh VS, Topcu M, Sebire G, Bodell A, Hill RS, Grant PE, Shugart YY, Imitola J, Khoury SJ, Guerrini R, Walsh CA (2004) Mutations in ARFGEF2 implicate vesicle trafficking in neural progenitor proliferation and migration in the human cerebral cortex. Nat Genet 36:69-76.

Shen X, Xu KF, Fan Q, Pacheco-Rodriguez G, Moss J, Vaughan M (2006) Association of brefeldin A-inhibited guanine nucleotide-exchange protein 2 (BIG2) with recycling endosomes during transferrin uptake. Proc Natl Acad Sci U S A 103:2635-2640.

Sol é G, Coupry I, Rooryck C, Guérineau E, Martins F, Devés S, Hubert C, 
Souakri N, Boute O, Marchal C, Faivre L, Landr é E, Debruxelles S, DieuxCoeslier A, Boulay C, Chassagnon S, Michel V, Routon MC, Toutain A, Philip N, Lacombe D, Villard L, Arveiler B, Goizet C (2009) Bilateral periventricular nodular heterotopia in France: frequency of mutations in FLNA, phenotypic heterogeneity and spectrum of mutations. J Neurol Neurosurg Psychiatry 80:1394-1398.

Vadlamudi RK, Li F, Adam L, Nguyen D, Ohta Y, Stossel TP, Kumar R (2002) Filamin is essential in actin cytoskeletal assembly mediated by p21-activated kinase 1. Nat Cell Biol 4:681-690.

Vicente-Manzanares M, Choi CK, Horwitz AR (2009) Integrins in cell migration-the actin connection. J Cell Sci 122:199-206.

Woo MS, Ohta Y, Rabinovitz I, Stossel TP, Blenis J (2004) Ribosomal S6 kinase (RSK) regulates phosphorylation of filamin A on an important regulatory site. Mol Cell Biol 24:3025-3035.
Xu W, Baribault H, Adamson ED (1998) Vinculin knockout results in heart and brain defects during embryonic development. Development 125:327-337.

Xu Y, Bismar TA, Su J, Xu B, Kristiansen G, Varga Z, Teng L, Ingber DE, Mammoto A, Kumar R, Alaoui-Jamali MA (2010) Filamin A regulates focal adhesion disassembly and suppresses breast cancer cell migration and invasion. J Exp Med 207:2421-2437.

Yamaji R, Adamik R, Takeda K, Togawa A, Pacheco-Rodriguez G, Ferrans VJ, Moss J, Vaughan M (2000) Identification and localization of two brefeldin A-inhibited guanine nucleotide-exchange proteins for ADPribosylation factors in a macromolecular complex. Proc Natl Acad Sci U S A 97:2567-2572.

Ziegler WH, Liddington RC, Critchley DR (2006) The structure and regulation of vinculin. Trends Cell Biol 16:453-460. 